\title{
Cervico-stylo-mandibular complex fracture: a critical review of literature along with a protocol to recognize and proposal of a new classification
}

\author{
Nanda Kishore Donepudi, Vinayak Gourish Naik, Kirthi Kumar Rai \\ Department of Oral and Maxillofacial Surgery, Bapuji Dental College \& Hospital, Davangere, India
}

\begin{abstract}
J Korean Assoc Oral Maxillofac Surg 2019;45:302-308)
Fractures of the styloid process of the temporal bone may occur with or without an obvious relation to trauma. The incidence of either isolated styloid process fracture or in combination with mandibular fractures is rare, and such occurrences are often misdiagnosed or neglected. A fractured styloid when displaced may impinge on adjacent vital structures, leading to neurological or vascular symptoms that vary according to the anatomical structure compressed. Styloid process fractures associated with atlas/ $\mathrm{C} 1$ fractures have also been rarely reported in the literature. In this review of literature, the majority of patients was treated conservatively, as few demonstrated the necessity of surgical intervention. There is a definitive need for a protocol to recognize and classify styloid fractures to plan for further treatment. The aim of this review was to achieve a comprehensive understanding of all types of styloid fractures, determine the clinical severity of symptoms, and to consider management and prognosis. In addition, a new classification of cervico-stylo-mandibular fractures is proposed based on important evidence in the literature regarding clinical and radiographic factors that might influence the treatment and prognosis of such fractures.
\end{abstract}

Key words: Styloid process fractures, Cervico-stylo-mandibular fractures, Classification

[paper submitted 2019. 1. 12 / revised 1st 2019. 4. 9, 2nd 2019. 5. 6 / accepted 2019. 5. 12]

\section{Introduction}

Though the actual incidence of styloid process (SP) fracture is high, failure to diagnose results in a smaller number of reported cases ${ }^{1}$. Incidence of styloid fracture concomitant with mandibular fractures is infrequent in occurrence $e^{2-5}$; due to the high variability of vague symptoms on presentation, such patients rarely report to a maxillofacial surgical unit ${ }^{6-8}$. The role of a maxillofacial surgeon is to prevent misdiagnosis or mismanagement of patients with SP fracture and to prevent long-term complications of traumatic styloid syndrome through appropriate treatment and post-operative review ${ }^{7,8}$. There is extremely sparse discussion on management of such fractures occurring along with mandibular fractures ${ }^{9}$. Rarely,

\footnotetext{
Vinayak Gourish Naik

Department of Oral and Maxillofacial Surgery, Bapuji Dental College \& Hospital, PO Box \#326, Davangere 577004, Karnataka, India

TEL: +91-8192-220335 FAX: +91-8192-220578

E-mail:drvinayakgn@gmail.com

ORCID: https://orcid.org/0000-0002-0323-8589

(c) This is an open-access article distributed under the terms of the Creative Commons Attribution Non-Commercial License (http://creativecommons.org/ licenses/by-nc/4.0/), which permits unrestricted non-commercial use, distribution, and reproduction in any medium, provided the original work is properly cited. Copyright (C) 2019 The Korean Association of Oral and Maxillofacial Surgeons. All rights reserved.
}

these styloid fractures may also be associated with fracture of the atlas ${ }^{10,11}$. The clinical dilemma regarding management of these cervico-stylo-mandibular fractures must be addressed by establishing a definitive treatment algorithm.

Recently, a few surgeons have focused on classification based on radiological imaging in temporal SP fractures ${ }^{12,13}$. A validated, comprehensive, and structured classification of cervico-stylo-mandibular fractures has not been proposed until now. An injury severity classification system would help surgeons in making decisions about the most appropriate treatment modalities and in including prognostic factors for clinically relevant patient outcomes ${ }^{14}$. The purpose of this study is to produce a clinical grading system based on the severity of symptoms by establishing an injury severity score grading system, taking into account management and prognosis. Currently, there is no efficient reproducible classification system that provides elements for treatment and prognosis. This study also aims to propose a new classification for cervico-stylo-mandibular fractures following a critical review of the literature. 


\section{Review of Literature}

A total of 30 case reports of SP fracture was critically reviewed from the existing literature. Among these, 18 involve isolated styloid fractures, 9 focus on stylo-mandibular fractures, and 3 involve fractures associated with the atlas/ C1. Isolated styloid fractures were excluded from the review. (Table 1)

Almost all the reported cases of stylo-mandibular fractures were managed conservatively by open reduction and internal fixation (ORIF) surgery with maxillomandibular fixation (MMF) for 2 to 4 weeks. Even in the case reported at our unit, we followed a closed reduction and MMF for 2 weeks. (Table 1, Fig. 1) The work of Gülicher and Gerlach ${ }^{15}$ reports a case of mandibular fracture that failed to be reduced due to fracture of the SP, which suggests the need for surgical removal of the fractured SP. Based on the evidence in the literature regarding clinical and radiographic factors, five parameters are considered to establish a severity score grading system.

Five epidemiological parameters (Table 2) are considered, with points given for fundamental elements in grading fracture types. These five parameters are (1) age, (2) energy of trauma, (3) symptoms, (4) association with other fractures, and (5) displacement.

For each of the five fundamental elements, a score of one or zero is given according to the presence or absence of the factor, respectively. Thus, factors can accumulate scores from zero to five points and are grouped into two possible types with increasing severity and complexity.

After verification of the presence of the elemental factors of the score classification (0-5), fractures are classified into two groups: Group I (with scores of 0-2) and Group II (with scores of 3-5).

Table 1. Stylo-mandibular fractures reported in the literature

\begin{tabular}{|c|c|c|c|}
\hline Study & Fractures (n) & Associated injuries & Treatment \\
\hline $\operatorname{Bird}^{2}(1954)$ & 1 Undisplaced & Bilateral condyle plus parasymphysis & MMF with eyelet wiring \\
\hline Haidar and Kalamchi ${ }^{3}(1980)$ & 1 Displaced and 1 undisplaced & $\begin{array}{l}\text { 1) Ipsilateral body; } \\
\text { 2) Ipsilateral condyle and bilateral } \\
\text { body fracture }\end{array}$ & Both MMF 4 wk \\
\hline $\begin{array}{l}\text { Raman and Samraj }{ }^{4}(1990) \& \\
\text { Gayathri et al. }{ }^{12}(2016)\end{array}$ & 1 Undisplaced & $\begin{array}{l}\text { Ipsilateral angle and contralateral } \\
\text { parasymphysis }\end{array}$ & MMF 4 wk \\
\hline Miloro $^{9}(1994)$ & 1 Displaced & $\begin{array}{l}\text { Ipsilateral parasymphysis and } \\
\text { contralateral angle }\end{array}$ & Conservative with ORIF of mandible \\
\hline Gülicher and Gerlach ${ }^{15}(2000)$ & $\begin{array}{l}1 \text { Undisplaced fracture with } \\
\text { ramus stuck behind SP }\end{array}$ & $\begin{array}{l}\text { Ipsilateral low condyle plus } \\
\text { contralateral body }\end{array}$ & ORIF of mandibular fractures \\
\hline Dubey et al. ${ }^{5}(2013)$ & 1 Undisplaced left SP & Ipsilateral condyle plus parasymphysis & Conservative with MMF 2 wk and ORIF \\
\hline $\begin{array}{l}\text { Mohammad and Wadhwania }{ }^{1} \\
\text { (2015) }\end{array}$ & 1 Undisplaced bilateral & $\begin{array}{l}\text { Bilateral SP fracture with left } \\
\text { condyle and right body }\end{array}$ & $\begin{array}{l}\text { Conservative with ORIF of mandibular } \\
\text { fractures }\end{array}$ \\
\hline This study (Fig. 1) & 1 Undisplaced bilateral & $\begin{array}{l}\text { Ipsilateral ramus and } \\
\text { contralateral parasymphysis }\end{array}$ & $\begin{array}{l}\text { ORIF and mandibular parasymphysis; } \\
\text { MMF } 2 \mathrm{wk}\end{array}$ \\
\hline
\end{tabular}

(MMF: maxillomandibular fixation, ORIF: open reduction and internal fixation, SP: styloid process)

Nanda Kishore Donepudi et al: Cervico-stylo-mandibular complex fracture: a critical review of literature along with a protocol to recognize and proposal of a new classification. $J$ Korean Assoc Oral Maxillofac Surg 2019
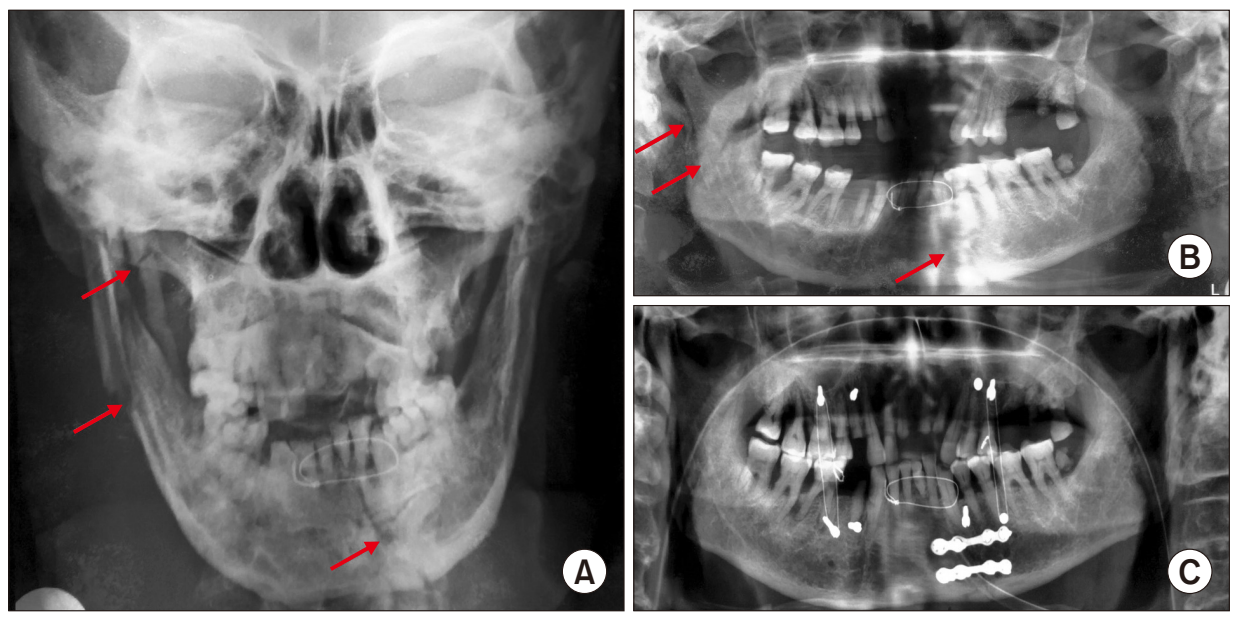

Fig. 1. A. Posteroanterior radiograph with arrows showing ipsilateral ramus fracture with styloid process fracture and contralateral parasymphysis fracture. B. Preoperative orthopantamogram (OPG) with arrows showing ipsilateral ramus fracture with styloid process fracture and contralateral parasymphysis fracture. C. Fifteen days postoperative OPG.

Nanda Kishore Donepudi et al: Cervico-stylomandibular complex fracture: a critical review of literature along with a protocol to recognize and proposal of a new classification. J Korean Assoc Oral Maxillofac Surg 2019 
For all patients over 40 years of age, one point is credited. Zero points are given to patients aged up to 40 years. An age factor of 40 years is specified because most of the literature confirms that elongation or lengthening of SP takes place after 40 years of age. Road traffic accidents, falls from heights, assault, crush injuries, and closed head trauma are categorized as high energy trauma, with one point assigned per incident. Minor incidents such as trauma due to repetitive yawning, coughing, impacted lower third molar removal ${ }^{16}$, and rolling over during sleep ${ }^{17}$ are examples of low energy trauma and are given a score of zero.

For patients presenting with mild symptoms, a score of zero is given. For severe symptoms of throat pain upon swallowing or neck motion, one point is credited. Similarly, one point is credited for SP fractures associated with other fractures such as mandible or atlas/C1 fractures, while zero points are given if there is no association with other fractures.

Table 2. Parameters used for scoring

\begin{tabular}{|c|c|c|c|}
\hline No. & Parameter & Criteria & Score \\
\hline \multirow[t]{2}{*}{1} & Age & $<40 \mathrm{yr}$ & 0 \\
\hline & & $>40 \mathrm{yr}$ & 1 \\
\hline \multirow[t]{2}{*}{2} & Energy of trauma & Low energy trauma & 0 \\
\hline & & High energy trauma & 1 \\
\hline \multirow[t]{2}{*}{3} & Symptoms & Mild to moderate & 0 \\
\hline & & Moderate to severe & 1 \\
\hline \multirow[t]{2}{*}{4} & Association with other fractures & Absent & 0 \\
\hline & & Present & 1 \\
\hline \multirow[t]{2}{*}{5} & Displacement & Absent & 0 \\
\hline & & Present & 1 \\
\hline
\end{tabular}

Nanda Kishore Donepudi et al: Cervico-stylo-mandibular complex fracture: a critical review of literature along with a protocol to recognize and proposal of a new classification. J Korean Assoc Oral Maxillofac Surg 2019
For fractures with displacement/deviation in radiographic assessment, one point is credited. For undisplaced fractures, zero points are given.

- Group I (scores of 0 to 2): Fractures with a maximum score of 2 points and relatively greater stability. They correspond to fractures in younger patients with low energy trauma or to fractures in patients over 40 years of age with no deviation or displacement of fractured styloid fragments. Group I fractures are usually managed conservatively and have a good prognosis.(Table 3)

- Group II (scores of 3 to 5): Fractures with a score of 3 to 5 points. Group II fractures correspond to fractures with displacement and to those associated with other fractures. Due to severity of symptoms, group II fractures are potentially unstable. They generally require surgical intervention with good stabilization, and prognosis is dependent on surgical removal of fractured styloid fragments along with treatment of associated fractures.(Table 3)

- Clinical presentation: Clinical examination and radiological investigation are essential in diagnosing and appropriately planning individual patient management. Trauma to the SP, although rarely discussed, can result in clinical symptoms of cervico-facial pain. Patients can have symptoms related to compression and irritation of cranial nerves (V, VII, IX, and $\mathrm{X}$ ), including facial pain while turning the head ${ }^{4}$, dysphagia $^{3}$, foreign body sensation, pain on protruding the tongue, temporomandibular joint dysfunction pain, change in voice, sensation of hypersalivation, tinnitus, or otalgia ${ }^{4}$. Sometimes, these symptoms are experienced with intermittent syncope. Intra-orally, symptoms would increase-ideally, for purposes

Table 3. Scores of reported cases

\begin{tabular}{|c|c|c|c|c|c|c|c|}
\hline No. & Study & Age & $\begin{array}{c}\text { Energy of } \\
\text { trauma }\end{array}$ & Symptoms & $\begin{array}{l}\text { Association with } \\
\text { other fractures }\end{array}$ & Displacement & $\begin{array}{l}\text { Total } \\
\text { score }\end{array}$ \\
\hline 1 & Haidar and Kalamchi ${ }^{3}(1980)$ & 0 & 1 & 1 & 1 & 0 & 3 \\
\hline 2 & Smith and Cherry ${ }^{7}(1988)$ & 0 & 1 & 1 & 1 & 1 & 4 \\
\hline 3 & Raman and Samraj ${ }^{4}$ (1990) & 0 & 1 & 1 & 1 & 0 & 3 \\
\hline 4 & Miloro $^{9}(1994)$ & 0 & 1 & 1 & 1 & 1 & 4 \\
\hline 5 & Gülicher and Gerlach ${ }^{15}(2000)$ & 1 & 1 & 1 & $\begin{array}{l}1 \text { (ramus stuck behind } \\
\text { styloid process) }\end{array}$ & 0 & 4 \\
\hline 6 & Klécha et al. ${ }^{23}$ (2008) & 1 & 1 & 0 & 1 & 1 & 4 \\
\hline 7 & Blythe et al. $^{8}(2009)$ & 1 & 0 & 0 & 0 & 0 & 1 \\
\hline 8 & Koivumäki et al. ${ }^{21}$ (2012) & 1 & 1 & 0 & 1 & 1 & 4 \\
\hline 9 & Dubey et al..$^{5}(2013)$ & 0 & 1 & 1 & 0 & 0 & 2 \\
\hline 10 & Mohammad and Wadhwania ${ }^{1}(2015)$ & 1 & 1 & 1 & 0 & 0 & 3 \\
\hline 11 & Mnari et al. ${ }^{11}(2016)$ & 0 & 1 & 1 & 1 (atlas/C1) & 1 & 4 \\
\hline 12 & Kermani et al. ${ }^{22}(2016)$ & 1 & 1 & 0 & 0 & 1 & 3 \\
\hline
\end{tabular}

Group I: Scores 0-2 (conservative treatment with a good prognosis), Group II: Scores 3-5 (conservative; +/- surgical removal if symptoms present or persistent).

Refer to Table 2 for the definition of scoring.

Nanda Kishore Donepudi et al: Cervico-stylo-mandibular complex fracture: a critical review of literature along with a protocol to recognize and proposal of a new classification. J Korean Assoc Oral Maxillofac Surg 2019 
of clinical diagnosis - on palpation. The clinical diagnosis of styloid fracture is always confirmed by imaging, which includes orthopantomograms, computed tomography (CT) scans $^{18}$, and conventional radiographs in lateral and posteroanterior views ${ }^{3,19}$. The use of CT or cone-beam CT (CBCT) scans with or without three-dimensional reconstruction is essential to establish the occurrence of a traumatic SP. Surgical removal of fractured styloid fragments is mandatory when the fracture is in close proximity to vital structures ${ }^{20-23}$. If pain is reproduced with simultaneous turning of a patient's head to the contralateral side and swallowing, then there should be a heightened index of suspicion for the presence of Eagle's syndrome ${ }^{21-23}$. Patients with chronic vague, generalized pain, with dysphagia, and with a sore throat and functional limitations in neck movement are ideal cases for surgical removal of fractured styloid fragments. If these cases are associated with mandibular fractures, ORIF with or without intermaxillary fixation (IMF) can be performed along with simultaneous removal of the fractured styloid fragment $\mathrm{t}^{22,23}$.

\section{Proposed Classification}

According to a critical review of the literature, SP fractures are classified based on the following.

- Based on displacement: Classifications include undisplaced fractures and displaced fractures.

- Based on the cause and grade of an impact: Classifications include intrinsic and extrinsic trauma.

- Based on fracture level: Classifications are high-level fractures and low-level fractures.

- Based on the direction of impact: Classifications are fractures from direct or indirect impact.

- Based on involvement: Classifications are unilateral or bilateral.

Although SP fractures are mentioned in previous literature, the terms "stylomandibular complex fractures" and "cervicostylomandibular fractures" are rarely used. We present herewith a comprehensive classification of stylo-mandibular fractures along with $\mathrm{C} 1$ fractures.

\section{Classification of Cervico-Stylo-Mandibular Fractures}

\section{Type A: Isolated SP fractures (Fig. 2)}

- Type A1: Undisplaced

- Type A2: Displaced medially with close proximity to vital structures

\section{Type B: Stylomandibular fractures (Fig. 3)}

- Type B1: Undisplaced

- Type B2: Displaced medially with close proximity to vital structures

3. Type C: Cervico-stylo-mandibular fractures (Fig. 4): fractures associated with fracture of the atlas/C1

- Type C1: Undisplaced

- Type C2: Displaced medially with close proximity to vital structures

Management of an isolated SP depends on the severity and amount of fractured SP displacement. Surgical removal may be necessary when either the SP is elongated or is severely displaced with close proximity to vital structures. In isolated SP fractures with mild discomfort, conservative treatment may be appropriate and sufficient. Conservative treatment in these cases includes analgesics and/or muscle relaxants with restriction of neck movement for several weeks to give the fracture site a chance to heal.

A clinical diagnosis of styloid fracture associated with mandibular fracture is difficult and misleading, because in many cases, a fractured SP may not be palpable at the tonsillar fossa region, and all the associated symptoms might be common to a mandibular angle fracture. Styloid fracture becomes evident only on orthopantamogram (OPG) and CT. For all intents and purposes, styloid fractures can be grouped into displaced or undisplaced.

For an undisplaced SP fracture, it is advisable to undergo OPG with an open mouth view. If a change in styloid position is revealed, then it is important to maintain IMF for 2 weeks following ORIF for mandibular fracture.

In cases of displaced SP fracture, it is always better to perform $\mathrm{CT}$. If the $\mathrm{CT}$ shows proximity of the fracture to vital structures, then surgical removal of the styloid fracture fragment along with ORIF of the mandible should be the treatment of choice. If the fractured SP is not close to vital structures, then ORIF of the mandible with or without IMF is initially advised. Excision of the fractured styloid fragment should be considered only if the fracture becomes symptomatic.

The work of Mnari et al. ${ }^{11}$ describes a unique case of a traumatic SP causing palsy of the lower cranial nerves. Similarly, Domenicucci et al. ${ }^{10}$ reports a case of post-traumatic Collet- 

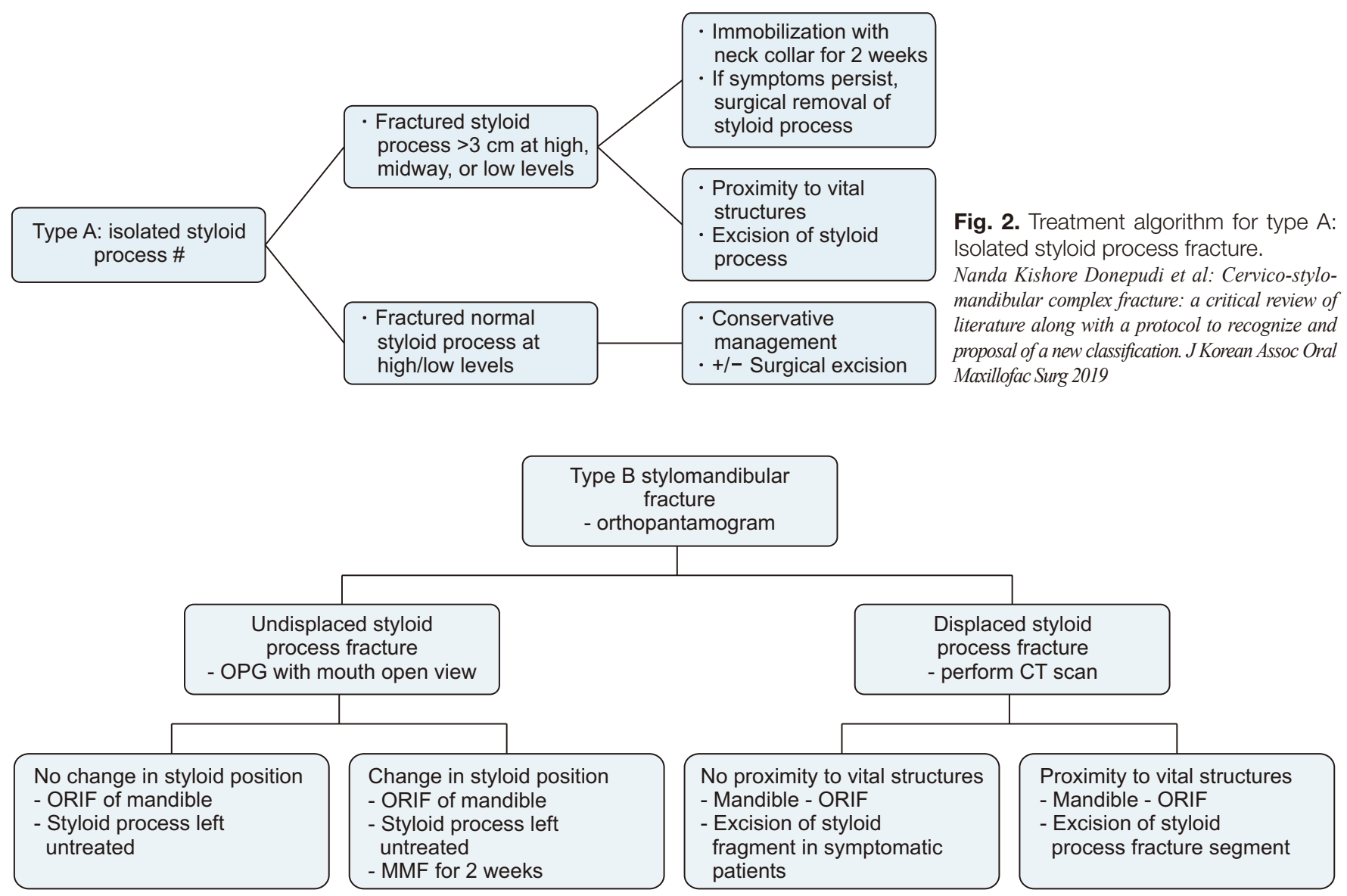

Fig. 3. Treatment algorithm for type B: Stylo-mandibular fractures. (OPG: orthopantamogram, CT: computed tomography, ORIF: open reduction and internal fixation, MMF: maxillomandibular fixation)

Nanda Kishore Donepudi et al: Cervico-stylo-mandibular complex fracture: a critical review of literature along with a protocol to recognize and proposal of a new classification. J Korean Assoc Oral Maxillofac Surg 2019

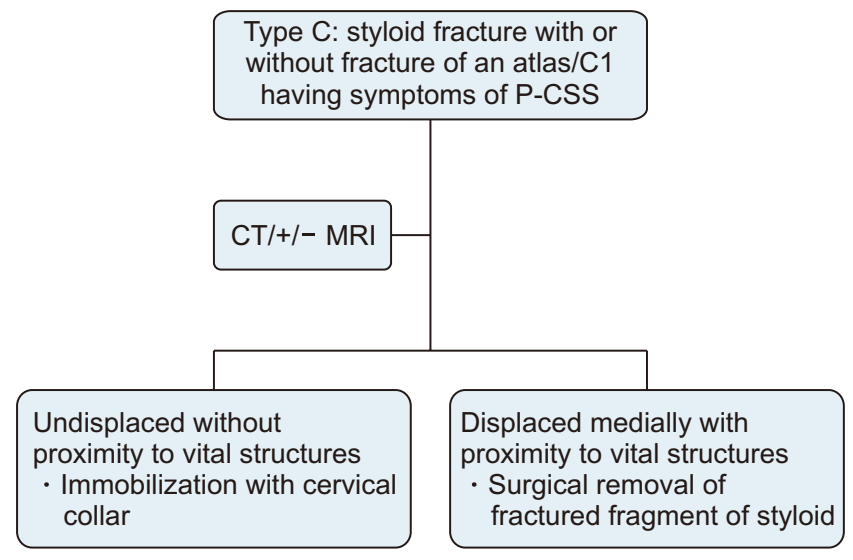

Fig. 4. Treatment algorithm for type C: Cervico-mandibular fractures or fractures associated with fracture of the atlas/C1. (P-CSS: post-traumatic Collet-Sicard syndrome, CT: computed tomography, MRI: magnetic resonance imaging)

Nanda Kishore Donepudi et al: Cervico-stylo-mandibular complex fracture: a critical review of literature along with a protocol to recognize and proposal of a new classification. J Korean Assoc Oral Maxillofac Surg 2019
Sicard syndrome. Collet-Sicard syndrome appearing secondary to a SP fracture is rare, and only a few case reports are available in the literature. Dettling et al. ${ }^{24}$ report a case with IX, X, and XI cranial nerve deficit with a 50-mm distance from the SP to the transverse process of the atlas on the fractured side. The narrow space between the transverse of the atlas and the SP make the lower cranial nerve vulnerable in the event of trauma. If the SP is abnormally displaced medially following trauma, then this space will be reduced, thereby increasing the likeliness of lower cranial nerve palsies. Therefore, cases of medially displaced SP must be treated with surgical excision of fractured styloid fragments. In contrast, undisplaced SP with no proximity to vital structures can be managed conservatively by immobilization of the cervical collar for 3 to 4 weeks. Surgical excision of the SP may be considered if a patient's symptoms persist.

These cases of styloid fractures with characteristics of Collet-Sicard syndrome may also be associated with atlas fracture. It is advisable to perform magnetic resonance imag- 
ing (MRI) along with CT for better depiction of soft tissues around the fractured SP. MRI also stands to reveal drooping and flabby aspects of the oropharyngeal wall on the fractured side or a deviated tongue related to paralysis of the IX and XII cranial nerves.

\section{Conclusion}

In conclusion, SP fractures may also be associated the cervico-mandibular region. It is important to determine the mode of treatment, either conservatively or surgically, based on the clinical severity of fractures. Surgery must be considered if a fractured fragment is found in close proximity to vital structures. The potential complications of post-traumatic styloid syndrome can be avoided with adequate treatment. Presentation of this classification would be easy to apply to guide prognosis and treatment for cervico-stylo-mandibular fractures. The main reasons for proposing a new classification system come both from our experience with clinical subjects involving these fractures and also based on the available literature. Consideration of the parameters presented here is grounded in the available scientific evidence. The empirical challenge of developing a severity classification system with the ability to assist in treatment and making prognoses is still a matter of debate.

\section{ORCID}

Nanda Kishore Donepudi, $h$ ttps://orcid.org/0000-0002-1406-3198 Vinayak Gourish Naik, https://orcid.org/0000-0002-0323-8589 Kirthi Kumar Rai, https://orcid.org/0000-0001-9745-177X

\section{Authors' Contributions}

N.K.D. and V.G.N. participated in data collection and wrote the manuscript. N.K.D., V.G.N., and K.K.R. participated in the study design and performed the statistical analysis. N.K.D., V.G.N., and K.K.R. participated in the study design and coordination and helped to draft the manuscript. All authors read and approved the final manuscript.

\section{Conflict of Interest}

No potential conflict of interest relevant to this article was reported.

\section{References}

1. Mohammad A, Wadhwania A. Bilateral fracture of styloid process. Int J Oral Health Med Res 2015;2:42-3.

2. Bird PD. Fracture of the styloid process secondary to fracture of the mandible. Br Dent J 1954;5:67.

3. Haidar A, Kalamchi S. Painful dysphagia due to fracture of the styloid process. Oral Surg Oral Med Oral Pathol 1980;49:5-6.

4. Raman U, Samraj T. Fracture of the styloid process associated with mandibular fracture. A case report. Int J Oral Maxillofac Surg 1990;19:350-1.

5. Dubey KN, Bajaj A, Kumar I. Fracture of the styloid process associated with the mandible fracture. Contemp Clin Dent 2013;4:1168.

6. Douglas BL, Huebsch RF. Atypical facial neuralgia resulting from fractured styloid process of the temporal bone. Oral Surg Oral Med Oral Pathol 1953;6:1199-201.

7. Smith GR, Cherry JE. Traumatic Eagle's syndrome: report of a case and review of the literature. J Oral Maxillofac Surg 1988;46:606-9.

8. Blythe JN, Matthews NS, Connor S. Eagle's syndrome after fracture of the elongated styloid process. Br J Oral Maxillofac Surg 2009;47:233-5.

9. Miloro M. Fracture of the styloid process: a case report and review of the literature. J Oral Maxillofac Surg 1994;52:1073-7.

10. Domenicucci M, Mancarella C, Dugoni ED, Ciappetta P, Paolo M. Post-traumatic Collet-Sicard syndrome: personal observation and review of the pertinent literature with clinical, radiologic and anatomic considerations. Eur Spine J 2015;24:663-70.

11. Mnari W, Kilani M, Harrathi K, Maatouk M, Koubaa J, Golli M. An unusual etiology of posttraumatic Collet-Sicard syndrome: a case report. Pan Afr Med J 2016;23:143.

12. Gayathri G, Elavenil P, Sasikala B, Pathumai M, Krishnakumar Raja VB. 'Stylo-mandibular complex' fracture from a maxillofacial surgeon's perspective--review of the literature and proposal of a management algorithm. Int J Oral Maxillofac Surg 2016;45:297303.

13. Panneerselvam E, Balasubramaniam S, Sharma AR, Murugadoss P, Chellappazham S, Raja VBK. Fractures of the stylomandibular complex: a prospective study proposing a classification and clinical protocol. J Oral Maxillofac Surg 2018;76:1734-44.

14. Martin JS, Marsh JL. Current classification of fractures. Rationale and utility. Radiol Clin North Am 1997;35:491-506.

15. Gülicher D, Gerlach KL. Failed closed reduction of a bifocal mandibular fracture because of dislocation of the mandibular ramus behind the styloid: case report. Br J Oral Maxillofac Surg 2000;38:280-2.

16. Raja K, Gopi G, Panneerselvam E, Ramamoorthy J, Doss GT, Sharma AR. Concomitant "ear bleed and styloid fracture": an unusual complication of impacted mandibular third molar removal. Craniomaxillofac Trauma Reconstr 2017;10:212-5.

17. Namiki H, Kobayashi T. Fracture of the styloid process in an elderly patient caused by rolling over during sleep: a case report. J Med Cases 2017;8:61-2.

18. Murtagh RD, Caracciolo JT, Fernandez G. CT findings associated with Eagle syndrome. AJNR Am J Neuroradiol 2001;22:1401-2.

19. Stafne EC, Hollinshead WH. Roentgenographic observations on the stylohyoid chain. Oral Surg Oral Med Oral Pathol 1962;15:1195200.

20. Fini G, Gasparini G, Filippini F, Becelli R, Marcotullio D. The long styloid process syndrome or Eagle's syndrome. J Craniomaxillofac Surg 2000;28:123-7.

21. Koivumäki A, Marinescu-Gava M, Järnstedt J, Sándor GK, Wolff J. Trauma induced eagle syndrome. Int J Oral Maxillofac Surg 2012;41:350-3.

22. Kermani H, Dehghani N, Aghdashi F, Esmaeelinejad M. Nonsyndromic isolated temporal bone styloid process fracture. Trauma 
Mon 2016;21:e24395.

23. Klécha A, Hafian H, Devauchelle B, Lefèvre B. A report of post-traumatic Eagle's syndrome. Int J Oral Maxillofac Surg 2008;37:970-2.

24. Dettling SD, Morscher MA, Masin JS, Adamczyk MJ. Cranial nerve IX and $\mathrm{X}$ impairment after a sports-related Jefferson (C1) fracture in a 16-year-old male: a case report. J Pediatr Orthop 2013;33:e23-7.
How to cite this article: Donepudi NK, Naik VG, Rai KK. Cervico-stylo-mandibular complex fracture: a critical review of literature along with a protocol to recognize and proposal of a new classification. J Korean Assoc Oral Maxillofac Surg 2019;45:302308. https://doi.org/10.5125/jkaoms.2019.45.6.302 